\title{
Spectroscopic and interferometric approach for differential rotation in massive fast rotators
}

\author{
Juan Zorec ${ }^{1}$, Yves Frémat ${ }^{2}$, Omar Delaa ${ }^{3}$, Armando Domiciano de \\ Souza $^{3}$, Philippe Stee ${ }^{3}$, Denis Mourard ${ }^{3}$, Lydia S. Cidale ${ }^{4}$, and \\ Christophe Martayan ${ }^{5}$ \\ ${ }^{1}$ Institut d'Astrophysique de Paris, UMR 7095 du CNRS, Univ.P\&MC, France (zorec@iap.fr) \\ ${ }^{2}$ Royal Observatory of Belgium, 3 av. Circulaire, 1180 Brussels, Belgium \\ ${ }^{3}$ Laboratoire Fizeau, UNS-OCA-CNRS UMR6203, Parc Valrose, 06108 Nice Cedex 02, France \\ ${ }^{4}$ Facultad de Ciencias Astronómicas y Geofísicas, Universidad Nacional de La Plata, Argentina \\ ${ }^{5}$ European Organization for Astronomical Research in the Southern Hemisphere, Chile
}

\begin{abstract}
The coupling between the convective region in the envelope and rotation can produce a surface latitudinal differential rotation that may induce changes of the stellar geometry and on the spectral line profiles that it may be scrutinized spectroscopically and by interferometry.
\end{abstract}

Keywords. stars: rotation, techniques: spectroscopic, techniques: interferometric

\section{Motivations}

Clement (1979) using a conservative distribution of the angular velocity, and Maeder et al. (2008), with a "shellular" distribution, have shown that in fast rotating earlytype stars the envelope layers laying from $1 / 3$ to $1 / 4$ of the stellar radius beneath the surface are unstable to convection. The baroclinic balance relation can then be solved by sketching the coupling between convection and rotation, assuming that the specific entropy is a function of the specific angular momentum $j, \Omega^{2}$ or the specific rotation energy $\epsilon_{\Omega}=\varpi^{2} \Omega^{2}$. Fig. 1 shows the curves of constant $\Omega$ for $S=S\left(\Omega^{2}\right)$ which can closely reproduce the differential rotation in the convective zone of the Sun, and $S=S\left(\epsilon_{\Omega}\right)$ that reproduces the differential rotation profiles obtained with models for radiative stars. According to Maeder (2009) the shape of the surface of a star with non-conservative rotation law can be described with:

$$
\Phi_{\mathrm{G}}(\theta)-\frac{1}{2} \Omega^{2}(\varpi, z) \varpi^{2}+\frac{1}{2} \int_{0}^{\theta} \varpi^{2}\left(\nabla \Omega^{2} . d \mathbf{s}\right)=\Phi_{\mathrm{G}}(0) .
$$

where $\Phi_{\mathrm{G}}(\theta)$ is the gravitational potential; $d \mathbf{s}$ in an arbitrary displacement over the stellar surface; $\varpi$ is the distance to rotation axis; $\Omega(\theta)$ is the angular velocity as a function on the co-latitude $\theta$. For a simplified Maunder velocity law $\Omega_{\odot}(\theta)=\Omega_{o}\left(1+\alpha \cos ^{2} \theta\right)$ the contours of stars shown in Fig. 1 were obtained for $\eta_{o}=\Omega_{o}^{2} / R_{\mathrm{e}}^{3} / G M=0.8$ and $\alpha=0.3$. We note that although $\eta_{o} \neq 1$ the stars approach the critical flattening $R_{\mathrm{e}} / R_{\mathrm{p}}=1.5$ obtained for the critical rotation in the Roche approximation of the surface equipotential.

\section{Spectroscopic and Interferometric signatures}

With a modified FASTROT (Frémat et al. 2005) code we can obtain synthetic line profiles produced by differentially rotating model atmospheres, where the gravity 


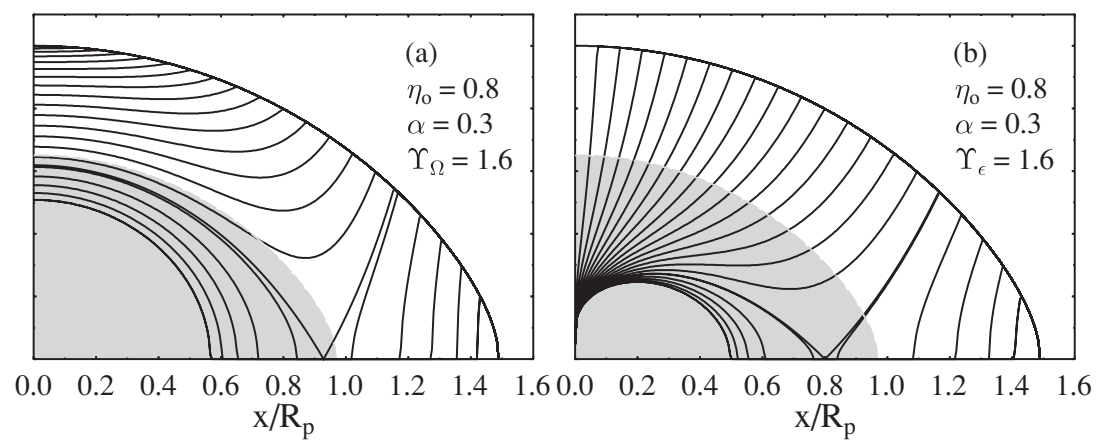

Figure 1. $\Omega(r, \theta)=$ constant curves. (a) $S=S\left(\Omega^{2}\right)$; (b) $S=S\left(\varpi^{2} \Omega^{2}\right)$. Solutions are not valid in the shaded zones. $\Upsilon_{\Omega, \epsilon}=\left[2 \alpha_{\Omega, \epsilon} G M\right] /\left[1-\alpha_{\Omega, \epsilon}\left(\Omega^{2}, \epsilon\right)\right] ; \alpha_{\Omega, \epsilon}=\left(1 / C_{\mathrm{P}}\right)\left[d S / d\left(\Omega^{2}, \epsilon\right)\right]$
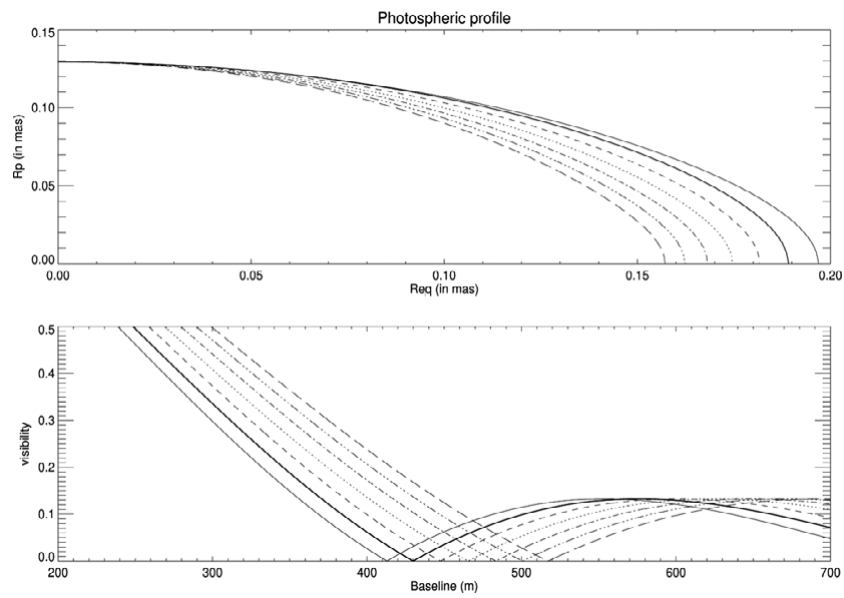

Figure 2. Apparent angular dimension of a B2V-type star seen at $d=300 \mathrm{pc}$ having a surface rotational parameter $\eta_{o}=0.7$ at the equator and deformed according to several values of the parameter $\alpha$ in the latitudinal differential rotation given by $\Omega_{\odot}(\theta)=\Omega_{o}\left(1+\alpha \cos ^{2} \theta\right)$. Visibility curves for the respective stellar deformations induced by the surface differential rotation.

darkening effect is calculated consistently with the shape changes of the star according to the adopted Maunder rotation law. The Fourier transform of the line profiles show that the ratio of the first two zeroes is a fairly nicely resolved quantity to estimate the differential rotation parameter $\alpha$. We have also calculated the visibility curves of differential-rotation deformed stars [see Fig. 2 as function of the baseline seen by the VEGA/CHARA for a B stars at 300 pc (Mourard et al. 2009)]. This calculation show that differences carried on the visibility curves in the visual spectral range by the geometrical deformations shown in the figure can be resolved with the VEGA/CHARA interferometric array. The combined spectroscopic and interferometric analysis could then lead to valuable constraints to the modeling of the surface differential rotation and the internal differential rotation beneath the surface.

\section{References}

Clement, M. J. 1979, ApJ, 230, 230

Frémat, Y., Zorec, J., Hubert, A.-M., \& Floquet, M. 2005, A\&\&A, 440, 305

Maeder, A., Georgy, C., \& Meynet, G. 2008, A\&A, 479, L37

Maeder, A. 2009, Physics, Formation and Evolution of Rotating Stars, (Springer)

Mourard, D., Clausse, J. M., Marcotto, A., Perraut, K. et al. 2009, A\&\&A, 508, 1073 


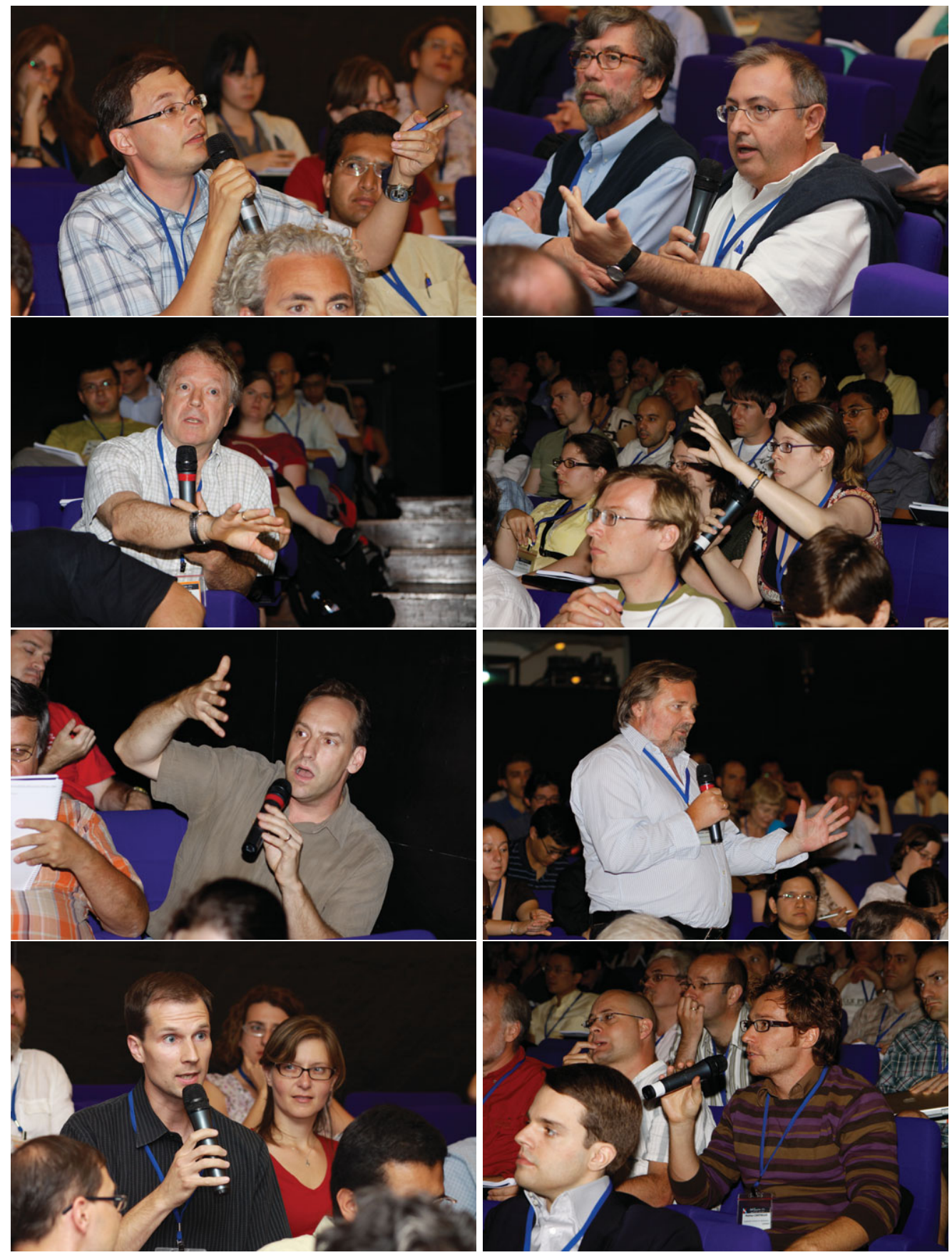

Questions in the audience. From left to right and top to bottom: Olivier Chesneau, Artemio Herrero (and Jean-Paul Zahn), Myron Smith, Véronique Petit, Marc Gagné, Norbert Langer, Christopher Tycner (and Erika Grundstrom), Matteo Cantiello (and Stéphane Mathis). 